\title{
Neuropil Threads of Alzheimer's Disease Show a Marked Alteration of the Normal Cytoskeleton
}

\author{
George Perry, ${ }^{1}$ Mitsuru Kawai, ${ }^{1}$ Massimo Tabaton, ${ }^{2}$ Michelle Onorato, ${ }^{1}$ Paul Mulvihill, ${ }^{1}$ Peggy Richey, ${ }^{1}$ Antonio \\ Morandi, ${ }^{2}$ Joe A. Connolly, ${ }^{2, a}$ and Pierluigi Gambetti ${ }^{1}$
}

'Division of Neuropathology, Institute of Pathology, Case Western Reserve University, Cleveland, Ohio 44106 and

2Department of Anatomy, Faculty of Medicine, Medical Sciences Building, University of Toronto, Toronto, Ontario,

Canada M5S 1 A8

\begin{abstract}
Abnormal neurites, neuropil threads, are a widespread and characteristic lesion of Alzheimer's disease likely to play a major role in the cognitive impairment of this disease. Contrary to normal neurites, neuropil threads contain straight and paired helical filaments that contain the microtubuleassociated protein tau and ubiquitin. It is not known whether these abnormal filaments are added to or replace the normal cytoskeleton. In this study, we examined the fine structure of neuropil threads and carried out a morphometric analysis of the neurofilaments and abnormal filaments contained in the neuropil threads by using an antiserum to tau and colloidal gold immuno-electron microscopy. Almost $70 \%$ of the neuropil threads contained straight or paired helical filaments with no neurofilaments. The total number of filaments in each neuropil thread remained essentially unchanged either when straight or paired helical filaments were present alone or when they coexisted either together or with neurofilaments. When the three types of filaments were expressed as a proportion of the total, a linear inverse correlation was found between neurofilaments and straight filaments as well as between straight and paired helical filaments. Approximately $10 \%$ of the neuropil threads were found to be myelinated axons. It is concluded that straight filaments are likely to replace neurofilaments, that they in turn might be replaced by paired helical filaments, and that this process occurs in axons as well as dendrites.
\end{abstract}

Braak and co-workers (Braak et al., 1986) introduced the term "neuropil thread" to identify the abnormal neurites present in the cerebral cortex of subjects with Alzheimer's disease. Subsequent studics have shown that neuropil threads are a common and extensive alteration in this disease (Joachim et al., 1987; Kowall and Kosik, 1987; Ihara, 1988). Contrary to normal neurites present in autopsy tissue, neuropil threads are immunostained by antibodies to tau or ubiquitin, which immunodecorate either paired helical filaments (PHFs) or 14-16-nm

Received Oct. 31, 1990; revised Jan. 4, 1991; accepted Jan. 11, 1991

We thank Sandy Bowen for manuscript preparation and Dr. Saeyd Amini for statistical consultation. This work was supported by NIH Grants AG08155, AG009287, and AG07552. G.P. is the recipient of NIH Research Career Development Award K04-AG00415. M.K. is a Fogarty Fellow, and A.M. is a fellow of the Britton Fund.

Correspondence should be addressed to George Perry, Ph.D., Division of Neuropathology, Institute of Pathology, Case Western Reserve University, 2085 Adelbert Road, Cleveland, OH 44106-4901.

${ }^{a}$ Deceased.

Copyright (C) 1991 Society for Neuroscience $0270-6474 / 91 / 111748-08 \$ 03.00 / 0$ nonhelical filaments termed "straight filaments" (Onorato et al., 1989; Tabaton et al., 1989). Because no other cellular component is recognized by the tau and ubiquitin antibodies in neuropil threads, the presence of paired helical and straight filaments is the distinctive feature of these abnormal neurites and is the one that confers the abnormal antigenic features to them. Neuropil threads have been found to be closely correlated with the distribution, severity, and antigenic characteristics of perikaryal neurofibrillary tangles. Neurofibrillary tangles and neuropil threads are considered to be expressions of a widespread alteration of the neuronal cytoskeleton, referred to as neurofibrillary pathology, which has been shown to play a significant role in the expression of dementia in Alzheimer's disease (Tabaton et al., 1989; McKee et al., 1990).

An important question concerning the neurofibrillary pathology in Alzheimer's disease is whether the formation of paired helical and straight filaments is associated with concurrent changes of the normal cytoskeleton. We addressed this question with an immuno-electron-microscopic, morphometric study of neuropil threads. Analyses of these data show that straight filaments and PHFs replace the normal cytoskeleton in neuropil threads. These findings are consistent with the hypotheses that, in neuropil threads, straight filaments form either by the gradual transformation of preexisting cytoskeletal structures or by the utilization of important cytoskeletal components so that the normal cytoskeleton cannot be maintained.

\section{Materials and Methods}

Tissue source. Hippocampi and the adjacent entorhinal cortices from two cases aged $60 \mathrm{yr}$ (case 1) and $71 \mathrm{yr}$ (case 2), both with a definite history of dementia and fulfilling the National Institute of Aging (NIA) consensus criteria for Alzheimer's disease (Khachaturian, 1985), and corresponding tissue from the same region of two age-matched control cases were used. Tissue was fixed with Bouin's solution.

Antibody characterization. Rabbit antiserum to bovine brain tau (Connolly and Kalnins, 1980) was immunoaffinity purified by chromatography on Sepharose column containing tau coupled by cyanogen bromide activation of Sepharose 4B. Tau was prepared by glycerol precipitation of a perchloric acid extract of bovine brain (Lindwall and Colc, 1984). The specific antibodies were eluted using $0.1 \mathrm{M}$ glycine $(\mathrm{pH}, 2.5)$ and standard procedures. Specificity of the affinity-purified antiserum to tau was proved by two means: (1) immunostaining of immunoblots prepared from $10 \%$ SDS-polyacrylamide gels (Laemmli, 1970 ) according to Towbin et al. (1979) containing either microtubule protein (Perry et al., 1982) or heat-stable microtubule-associated proteins (Herzog and Weber, 1978) and (2) immunoabsorption of the affinity-purified serum $(0.25 \mu \mathrm{g} / \mathrm{ml})$ performed with bovine brain tau (50 $\mu \mathrm{g} / \mathrm{ml}$; Lindwall and Cole, 1984) for $16 \mathrm{hr}$ at $4^{\circ} \mathrm{C}$. On immunoblots, the crude antiserum to tau recognized tau-protein bands and microtubule associated protein 2 (MAP2) and showed some background, but the 


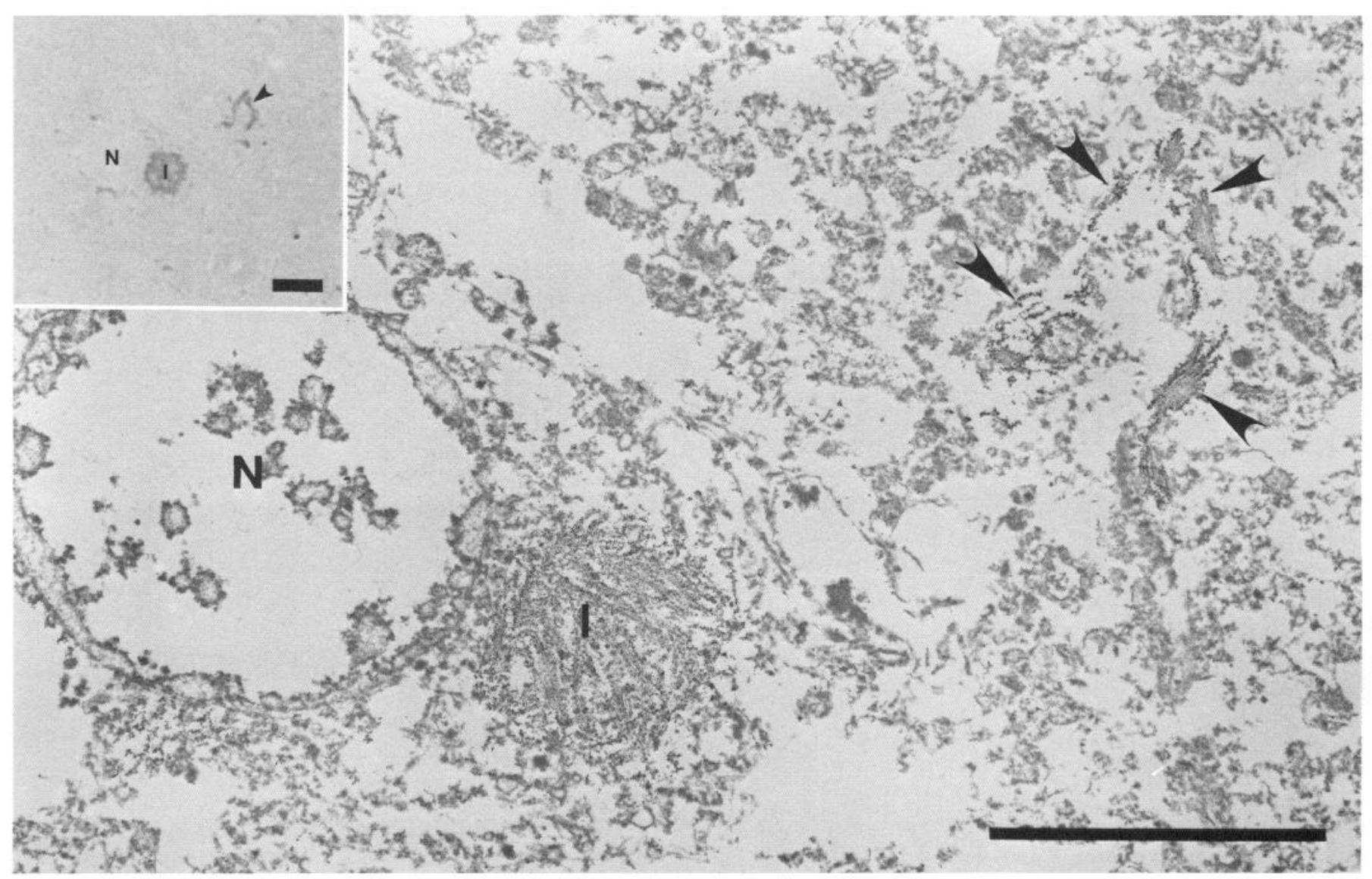

Figure 1. The ultrastructural correlate of the immunostained neuropil thread was demonstrated by correlative light and electron microscopy of the same specimen. The inset is a phase-contrast light micrograph of a 1- $\mu$ m-thick plastic section with an immunostained neuropil thread (arrowhead) adjacent to a neuron containing an unusual juxtanuclear $(N)$ immunostained inclusion $(I)$. The next section $(60 \mathrm{~nm})$ was examined in the electron microscope. The same nucleus $(N)$, immunodecorated inclusion $(I)$, and immunodecorated neuropil thread (arrowheads) were again found. Antiserum to tau was used. Scale bars, $5 \mu \mathrm{m}$.

immunoaffinity-purified antibodies were selective for tau, showing only a weak reaction for MAP2 and no background (not shown). Absorption with purified tau abolished the reaction of the immunoaffinity-purified antiserum with tau bands in immunoblots and neurofibrillary pathology in tissue sections.

Light- and electron-microscopic immunocytochemistry. Immunocytochemistry for the light microscope was performed by the peroxidaseanti-peroxidase method (Sternberger, 1986) on tissue from all four cases after embedding in paraffin and sectioning at $6 \mu \mathrm{m}$. Vibratome (Oxford) sections $(60 \mu \mathrm{m})$ for immuno-electron microscopy were prepared from the two cases of Alzheimer's disease without prior embedding of the tissue. Vibratome sections were treated with $0.5 \%$ Triton X-100 in 0.15 $\mathrm{M} \mathrm{NaCl}$ and $50 \mathrm{~mm}$ Tris- $\mathrm{HCl}(\mathrm{pH}, 7.6)$ at room temperature for $10 \mathrm{~min}$, before incubation with the primary antibody for $2 \mathrm{~d}$ at $4^{\circ} \mathrm{C}$. The sections were then incubated for $2-3 \mathrm{~d}$ with colloidal gold $(\simeq 17 \mathrm{~nm})$ complexed to affinity-purified anti-rabbit IgG (Cappel Laboratories; DeMey, 1983). Sections were subsequently fixed with $2.5 \%$ glutaraldehyde in $0.1 \mathrm{M}$ phosphate buffer $(\mathrm{pH}, 7.2)$ for $2 \mathrm{hr}$ followed by postfixation in $2 \% \mathrm{OsO}_{4}$ and flat embedding in Spurr's medium. Normal rabbit serum was substituted for primary antibody in order to evaluate background staining. Sections were viewed at $60 \mathrm{kV}$ in a JEOL $100 \mathrm{CX}$ electron microscope.

Immuno-electron microscopy and quantitative analysis. Only immunodecorated neurites were considered neuropil threads and examined in both Alzheimer's cases. The neuropil threads were positively identified at the ultrastructural level with comparative light- and electron-microscopic examination of consecutive $1-\mu \mathrm{m}$-thick and $60-\mathrm{nm}$ thick sections, respectively, obtained from immunogold-decorated tissue. As shown in Figure 1, the immunodecorated neuropil thread observed in a 1- $\mu$ m-thick section under the light microscope was then identified using tissue landmarks in the consecutive 60 -nm-thick section viewed with the electron microscope. There was a close correlation between neuropil threads detected at light and electron microscopy because even neuropil threads with only three immunodecorated filaments (not shown) were visible in the light microscope. For this reason, control cases that did not show any neuropil thread at light-microscopic examination following immunostaining with the antiserum to tau were not studied with immuno-electron microscopy.

Quantitative analyses were carried out only in Alzheimer case 2. Sections were scanned at 20,000 magnification so that, if even only one labeled filament was identified, the structure could be classified as a neuropil thread. Special attention was paid to exclude any filament bundles near amyloid fibrils and neuronal cell bodies. Micrographs at 20,000 and 40,000 magnification were made of each of the 205 neuropil threads found in the $0.44 \mu \mathrm{m}^{2}$ examined. The filament type could be determined unambiguously in 168 neuropil threads. The primary reason for excluding neurites was poor micrograph quality. Many neuropil threads were examined in tangential or cross sections, which allowed the evaluation of all the filaments. In neuropil threads cut longitudinally, only the filaments in the plane of sections could be evaluated. Moreover, in these threads the same filament might have been scored twice when coming in and out of the plane of section, yet even in the latter neurites, the bias should affect only the absolute number of filaments and not the type representative because all filament types should be equally affected. The distribution between tangentially and longitudinally cut neurites was entirely random and thus did not introduce any bias in the representation of each of the three types of filaments examined (see Results).

\section{Results}

The antiserum to tau-immunostained neuropil threads, neurofibrillary tangles, and dystrophic neurites in senile plaques 
Figure 2. Immuno-electron micrograph of a myelinated neuropil thread in which neurofilaments predominate. Immunodecoration is associated with the straight filaments (arrows), while the abundant neurofilaments (arrowheads) contain few if any gold particles. Antiserum to tau was used. Scale bar, 500 $\mathrm{nm}$.

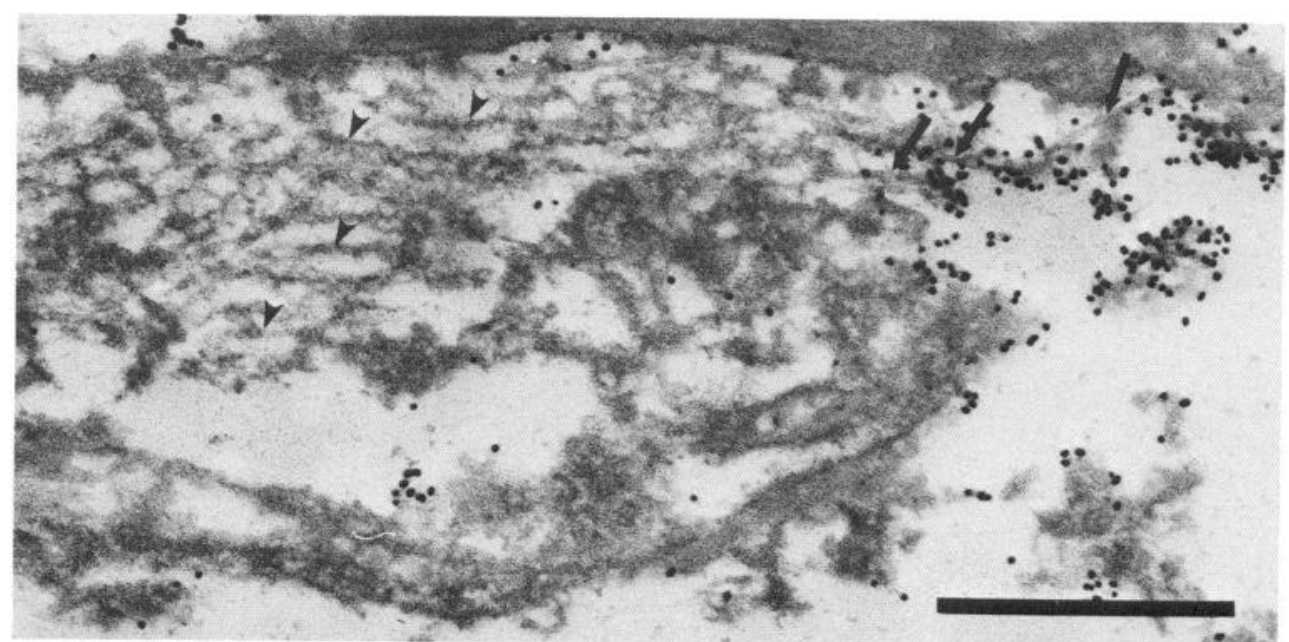

within the gray matter of both Alzheimer's disease cases was examined. The antiserum did not react with any structures in sections prepared from the same brain regions of the two agematched control cases (not shown), which were not investigated further (see below). Electron-microscopic examination of the immunodecorated tissue showed that the antibodies recognized both paired helical and straight filaments present in neurofibrillary tangles (not shown). In addition, immunodecoration of filaments organized in "bundles" was also prominent. These filamentous bundles were smaller and more irregular and elongated than neurofibrillary tangles. Moreover, they lacked lipofuscin and differed from dystrophic neurites of senile plaques, because they were not adjacent to amyloid fibers. Some of the immunostained filamentous bundles seen in the electron microscope were contained in processes that were clearly myelin- ated, in which case the decoration on some filaments depended on their proximity to an opening in the myelin (see, e.g., Fig. 3 ). The filamentous bundles were positively identified as neuropil threads with combined light- and electron-microscopic examination of $1-\mu \mathrm{m}$-thick and $60-\mathrm{nm}$-thick consecutive sections, as demonstrated in Figure 1, where the immunodecorated neuropil thread observed in a $1-\mu \mathrm{m}$-thick section under the light microscope can be recognized using tissue landmarks in the consecutive thin section viewed with the electron microscope.

Electron-microscopic analysis showed that neuropil threads contained three major types of filaments: neurofilaments (Fig. 2), straight filaments (Fig. 3), and PHFs (Fig. 4). In addition, two intermediate types were occasionally observed, one displaying an equivocal helicity consisting of periodic constrictions in diameter (Fig. 5), different from that of PHFs, and another

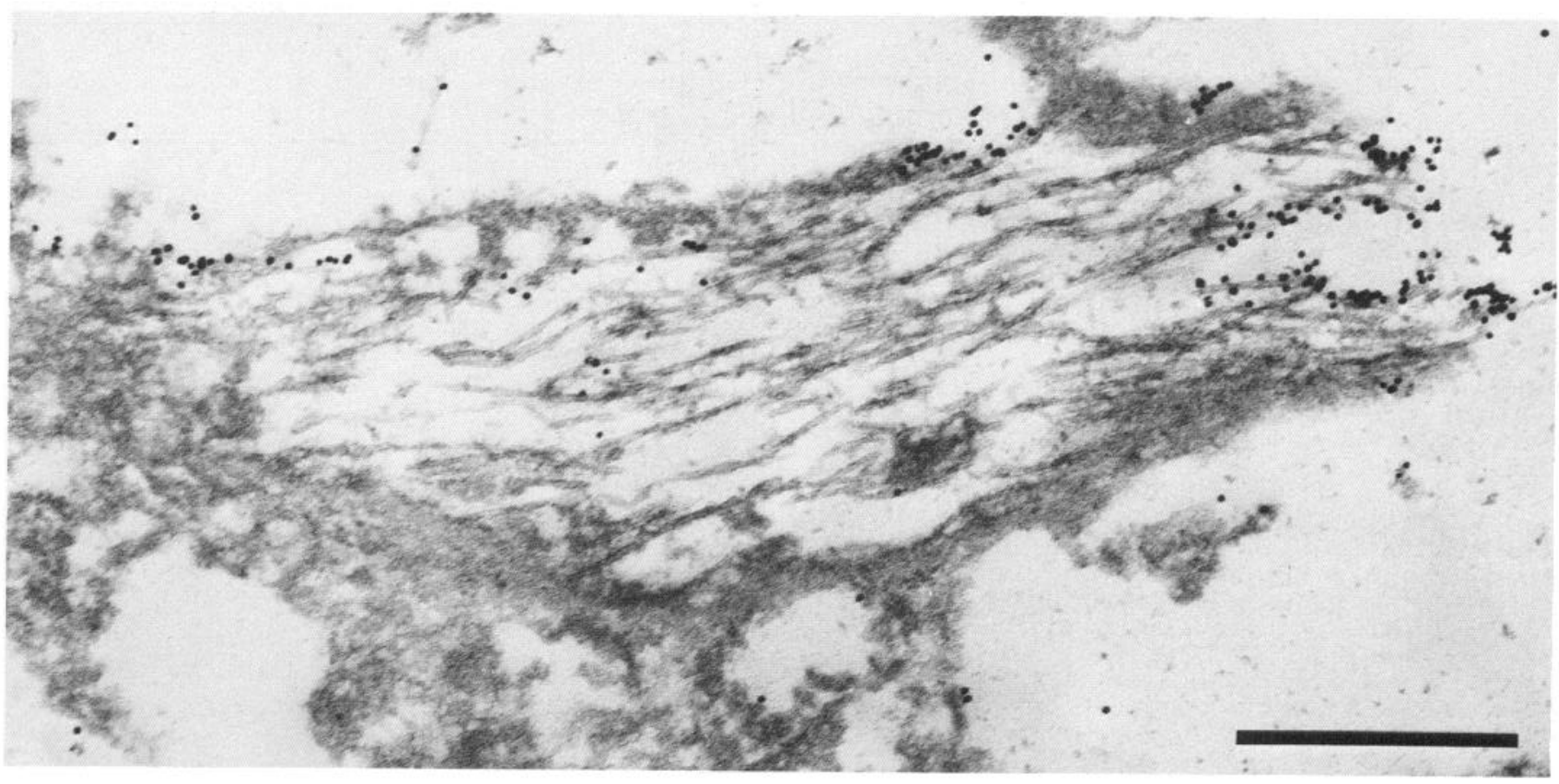

Figure 3. Over $10 \%$ of neuropil threads are myelinated. The hetergeneous decoration of straight filaments is probably due to poor penetration of gold particles through the sheath, presumably myelin, covering the filament bundles. Antiserum to tau was used. Scale bar, $500 \mathrm{~nm}$. 


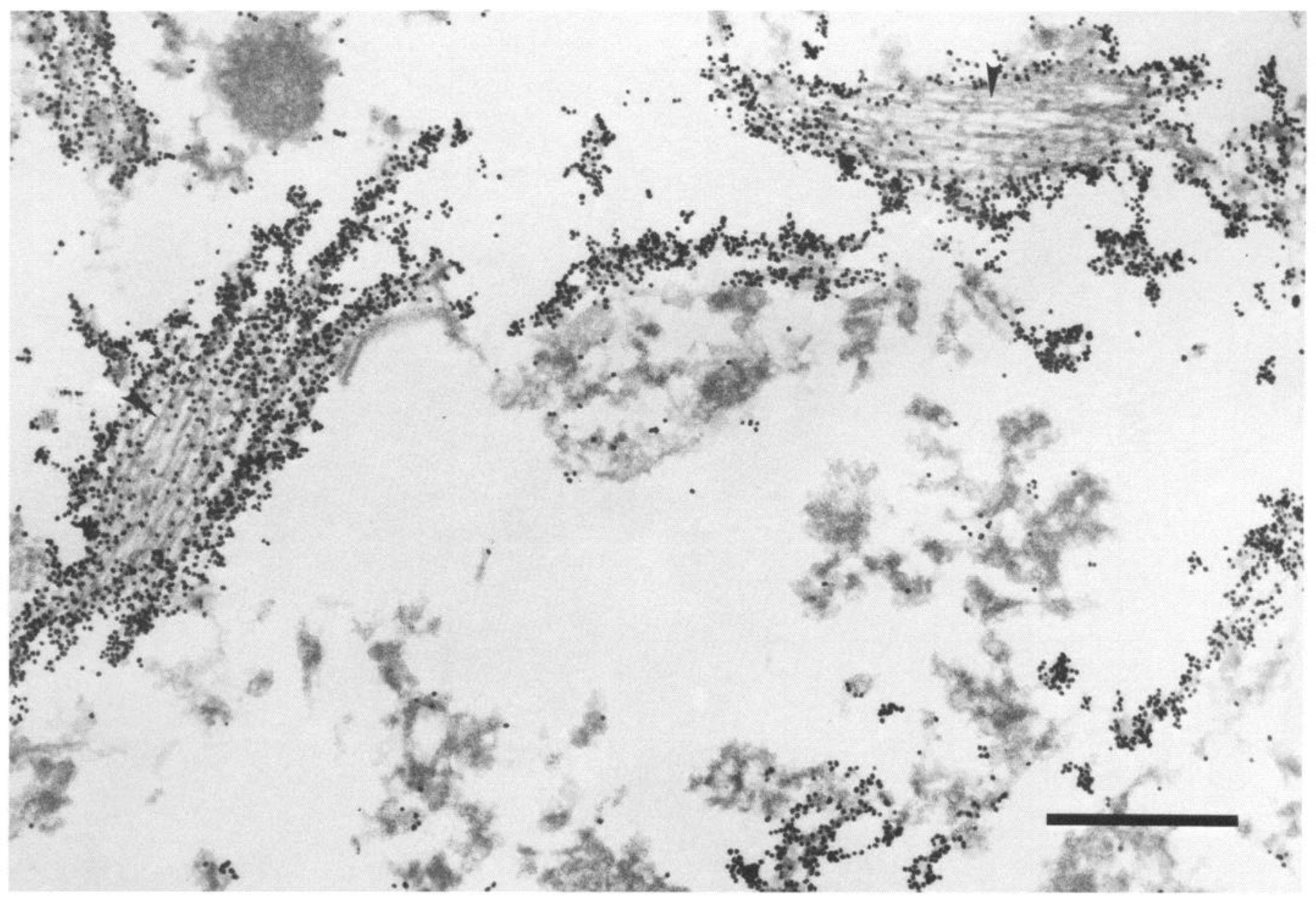

Figure 4. When viewed at high magnification, the neuropil thread shown in Figure 1 demonstrates abundant PHFs (arrowheads; see text). Antiserum to tau was used. Scale bar, $500 \mathrm{~nm}$.

displaying a surface smoother than that of neurofilaments and yet having side arms and the size of neurofilaments (Fig. 6).

Morphometric analysis was carried out in neuropil threads to determine both in absolute as well as in relative number the prevalence and possible interrelationships of neurofilaments, straight filaments, and PHFs in neuropil threads. The few intermediate types of filaments encountered were counted according to their dominant feature.

In almost $70 \%$ of the neuropil threads, straight filaments, and/ or PHFs were present alone, without neurofilaments (Table 1). PHFs alone were seen only in the absence of neurofilaments (Table 1). The total numbers of filaments either when straight filaments and PHFs were present alone or when they coexisted with the neurofilaments were not significantly different ( $p=$ 0.44 , Kruskal-Wallis; Table 1), suggesting that the addition of one filament type was associated with the loss of another; for example, neurofilaments were negatively correlated with straight filaments $(r=0.33 ; p=0.015)$. When the three types of filaments were expressed as a proportion of the total, there was a linear inverse correlation between presence of neurofilaments and straight filaments on the one hand and between presence of straight filaments and paired PHFs on the other (Fig. 7). These findings indicate that straight filaments and PHFs replace, rather than are added to, the neurofilaments in neuropil threads.

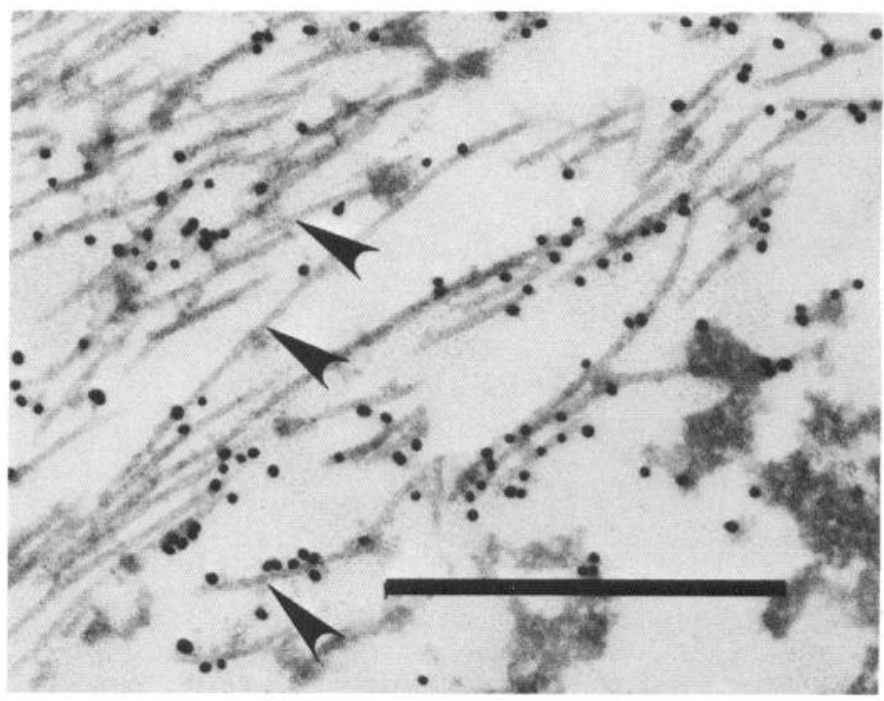

Figure 5. Helical filaments have a morphology intermediate between straight filaments and PHFs (see Results). Arrowheads indicate some of the filaments showing periodic constriction in diameter. Antiserum to tau was used. Scale bar, $500 \mathrm{~nm}$. 

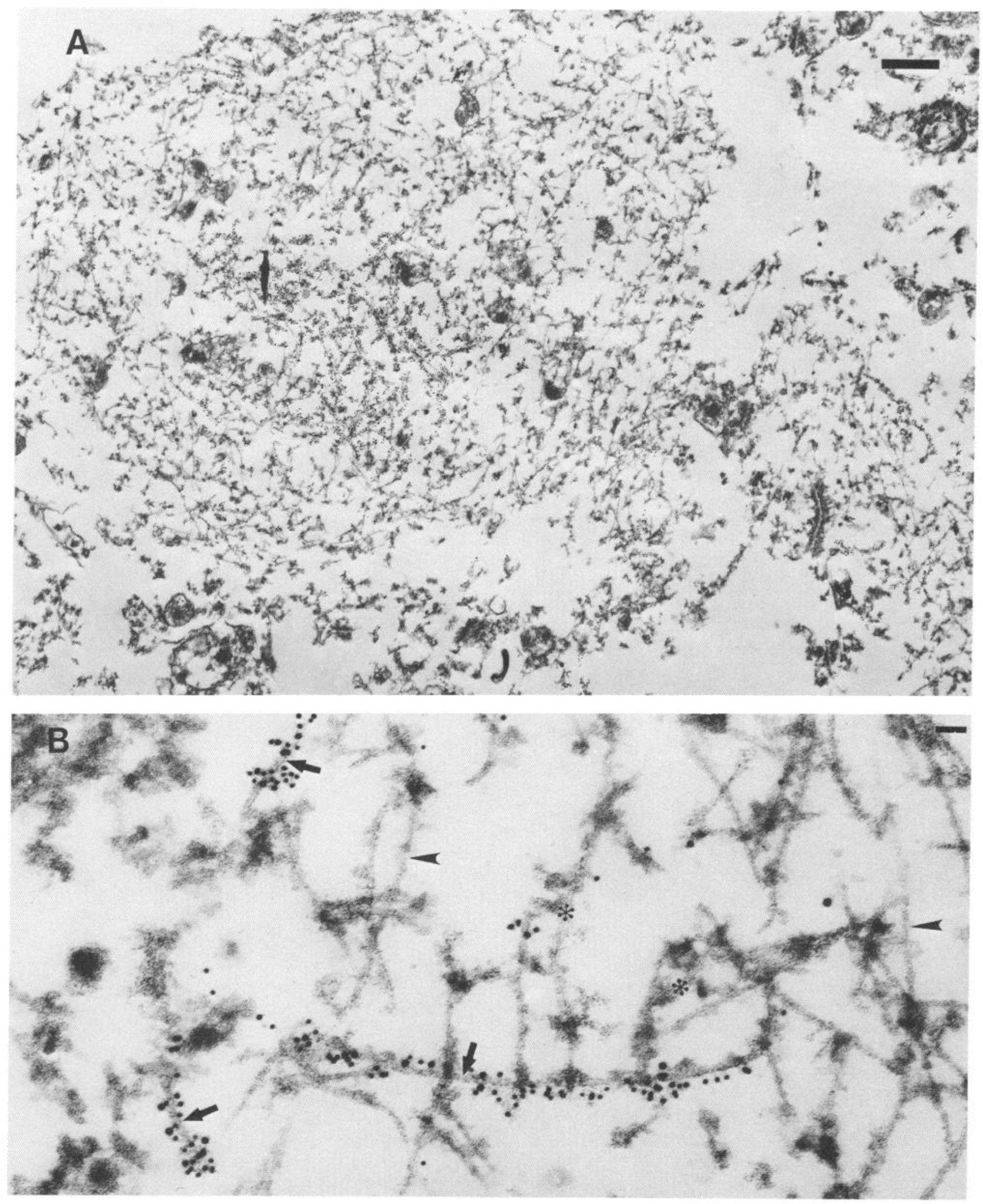

Figure 6. Straight filaments (14.6 $\pm 0.4-\mathrm{nm}$ diameter) are appreciated in a neuropil thread in which they coexist with neurofilaments. $B$, Viewed at higher magnification, the intense decoration of the relatively more rigid and smoother-surfaced decorated straight filaments (arrows) is appreciated as distinct from the neurofilaments $(11.3 \pm 0.1$-nm diameter; arrowheads). Some neurofilaments have less prominent side arms and look similar to straight filaments, though they were not decorated (*). Antiserum to tau was used. Scale bars: $A, 500 \mathrm{~nm} ; B, 60 \mathrm{~nm}$. 


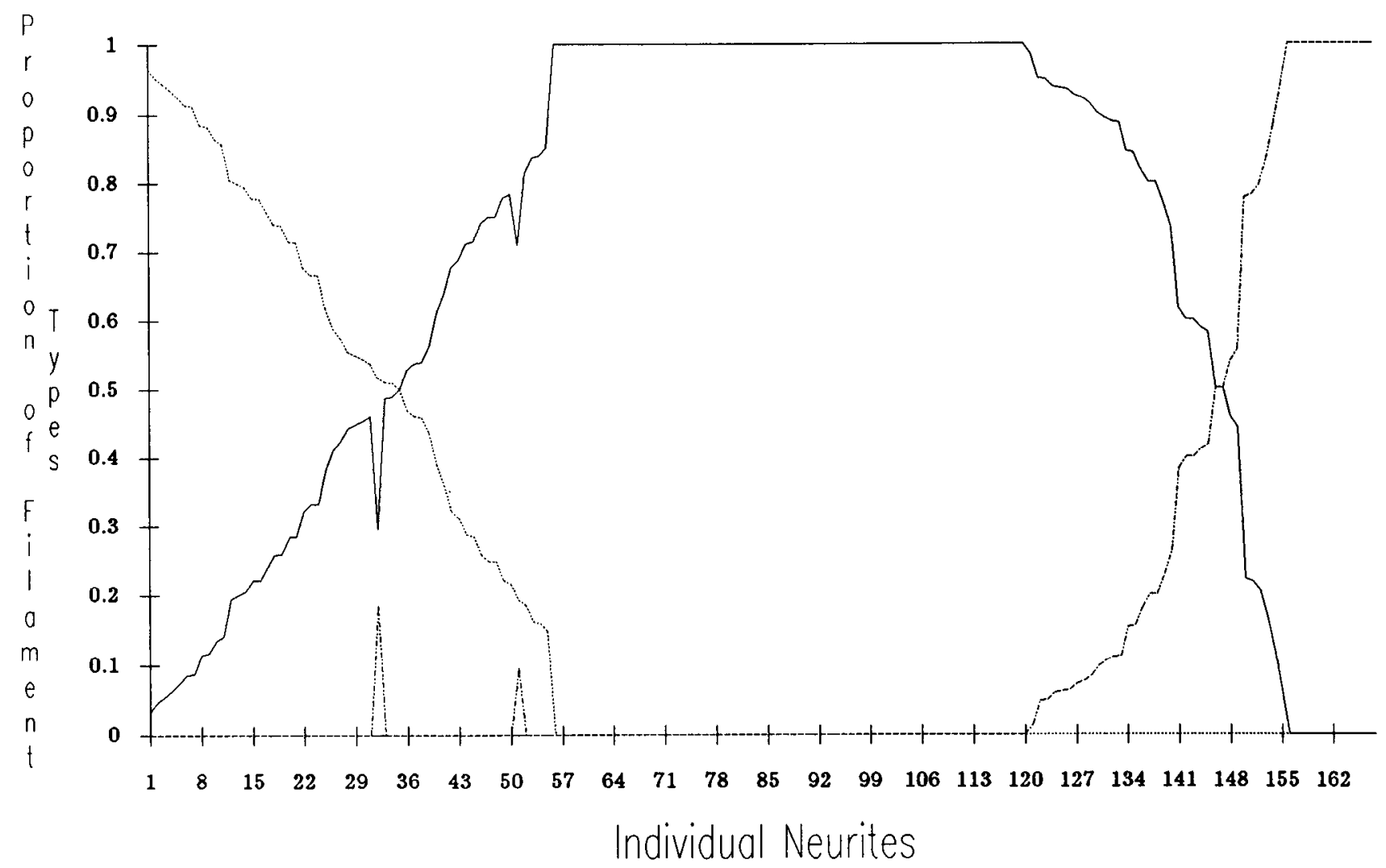

Figure 7. Proportion of filament types in individual neuropil threads for an area of $0.44 \mathrm{~mm}^{2}$ in case 2 . The neurites have been arranged according to decreasing neurofilaments, in their absence in increasing PHFs, and in the absence of both, in between. Dotted line, neurofilaments; solid line, straight filaments; dot-dashed lines, PHFs.

Although lacking PHFs, the 17 myelinated neuropil threads showed a similar inverse correlation between presence of neurofilaments and straight filaments $(r=0.76 ; p<0.01)$.

\section{Discussion}

Despite the obvious limitations imposed on this study by the suboptimal preservation of the brain parenchyma due to postmortem autolysis, the present findings demonstrate that, in most neuropil threads of Alzheimer's disease, abnormal filaments, which include straight filaments and PHFs, replace the neurofilaments. Thus, in neuropil thrcads, the presence of straight filaments and PHFs is associated with a profound alteration of the preexisting cytoskeleton. A similar inverse correlation be- tween PHFs and microtubules was observed (Ellisman et al., 1987; Metuzals et al., 1987, 1988) in a study of brain tissue obtained at biopsy, which, however, did not include morphometric analysis. Although microtubules could not be examined in our tissue samples, it is likely that straight filaments and PHFs replace not only neurofilaments but also microtubules in neuropil threads and that this profound alteration of the neuronal cytoskeleton is present also in the perikaryon of neurons containing neurofibrillary tangles. Thus, this study underlines the severe cytoskeletal alterations that neurons may undergo in Alzheimer's disease.

The modalities by which straight filaments and PHFs replace neurofilaments and possibly other cytoskeletal components such

Table 1. Neuropil threads divided by the constituent filaments and the number of various filament types in each

\begin{tabular}{llllll}
$\begin{array}{l}\text { Constituent } \\
\text { filaments }\end{array}$ & $\begin{array}{l}\text { Number of } \\
\text { neuropil } \\
\text { threads }\end{array}$ & NF & SF & PHF & Total \\
\hline NF and SF & $53(32 \%)$ & $17.1 \pm 1.6$ & $15.2 \pm 2.9$ & - & $32.3 \pm 3.7$ \\
NF and PHF & 0 & - & - & - & - \\
NF, SF, and PHF & $2(1 \%)$ & $10.0 \pm 5.7$ & $15.0 \pm 9.9$ & $4.0 \pm 1.4$ & $29.0 \pm 2.8$ \\
SF & $65(39 \%)$ & - & $36.6 \pm 3.9$ & - & $36.6 \pm 3.9$ \\
SF and PHF & $35(21 \%)$ & - & $33.5 \pm 6.7$ & $14.7 \pm 3.4$ & $48.3 \pm 7.8$ \\
PHF & $13(8 \%)$ & - & - & $42.2 \pm 16.4$ & $42.2 \pm 16.4$ \\
\hline
\end{tabular}

Values are mean \pm standard error of the mean. NF, neurofilaments; SF, straight filaments. 
as microtubules remain to be established. The simplest interpretation of the present data is that straight filaments form either directly by transformation of neurofilaments or indirectly by the utilization of neurofilament or other cytoskeletal constituents so that the normal cytoskeleton cannot be maintained. The formation of straight filaments does not necessarily take place in the neurites because they could migrate to the neurites from the cell body by axonal or dendritic transport. Three lines of evidence support the origin of straight filaments from the normal cytoskeleton. First, as our findings show, there is an inverse relation between neurofilaments and straight filaments, and only few neurites contain similar ratios of these two types of filaments. Second, straight filaments share epitopes with the normal cytoskeleton suggesting that these two structures have common components. A third line of evidence comes from the findings that straight filaments of Alzheimer's disease are insoluble in ionic detergents (Perry et al., 1987) and that they contain epitopes of ubiquitin (Manetto et al., 1988). In an experimental model, it was reported that abnormal ubiquitin is associated with the filaments of the Mallory bodies and serves as a marker for the intermediate filaments destined to be incorporated into the inclusion (Ohta et al., 1988). Ubiquitination was associated with an increase in diameter, a decrease in solubility, and a change of antigenic characteristics of the cytokeratin intermediate filaments in Mallory bodies. These changes are similar to the relationship we note here between neurofilaments and straight filaments. Should this be the case, then straight filaments of neuropil threads would derive from preexisting neurofilaments through an extensive transformation in which the acquisition of tau and ubiquitin and change in size, solubility, and antigenic characteristics would be some of the steps.

PHFs alone were never seen concurrently with neurofilaments in the same neuropil threads; they were seen either alone or in the presence of straight filaments. This finding indicates that formation of PHFs occurs when the preexisting cytoskeleton is markedly altered, and therefore it is a late event in the pathogenesis of the neuropil thread. Concerning the mode of formation of PHFs, one may apply the same rationale used above to postulate that PHFs derive directly or indirectly from the straight filaments. Consistent with this hypothesis is the finding that the presence of straight filaments is believed to precede the appearance of PHFs in the formation of neurofibrillary tangles (Tabaton et al., 1988). In the case of PHFs, however, these abnormal filaments might well form independently from straight filaments in a subpopulation of neurites.

Approximately $10 \%$ of the neuropil threads were found to be myelinated (Figs. 2, 3). This may be an underestimation of the actual number of axons that have become neuropil threads because not all gray matter axons are myelinated. The finding that a significant number of neuropil threads are axons contrasts with the suggestion that they form solely from dendritic sprouting (Braak and Braak, 1988; Ihara, 1988; McKee et al., 1989) but is consistent with the straight filaments shown in myelinated axons of biopsy specimens (Metuzals et al., 1987).

In conclusion, though the origin of the straight filaments and PHFs present in neuropil threads must remain speculative, the present study definitely shows that the presence of these abnormal filaments in neuropil threads is associated with severe decrease or disappearance of neurofilaments. On the basis of the present findings, it can be postulated that straight filaments and PHFs are the only structures related to the cytoskeleton left in most neuropil threads of Alzheimer's disease. Such an alteration of the cytoskeleton is likely to cause a marked reduction of functions such as axonal and dendritic transport.

\section{References}

Braak H, Braak E (1988) Neuropil threads occur in dendritcs of tanglcbearing nerve cells. Neuropathol Appl Neurobiol 14:39-44.

Braak H, Braak E, Grundke-Iqbal I, Iqbal K (1986) Occurrence of neuropil threads in the senile human brain and in Alzheimer's disease: a third location of paired helical filaments outside of neurofibrillary tangles and neuritic plaques. Neurosci Lett 65:351-355.

Connolly JA, Kalnins VI (1980) The distribution of tau and HMW microtubule-associated proteins in different cell types. Exp Cell Res 127:341-350

DeMey J (1983) Colloidal gold probes in immunocytochemistry. In: Immunocytochemistry (Polak JM, Van Noorden S, eds), pp 82-112. Briston, UK: Wright.

Ellisman M, Ranganathan R, Deerinck T, Young S, Terry R. Mirra S (1987) Neuronal fibrillar cytoskeleton and endomembrane system organization in Alzheimer's disease. In: Alterations in the neuronal cytoskeleton in Alzheimer disease (Perry G, ed), pp 61-73. New York: Plenum.

Herzog W, Weber K (1978) Fractionation of brain microtubule-associated proteins. Eur J Biochem 92:1-8.

Ihara $Y$ (1988) Massive somatodendritic sprouting of cortical neurons in Alzheimer's disease. Brain Res 459:138-144.

Joachim CL, Morris JH, Selkoe DJ, Kosik KS (1987) Tau epitopes are incorporatcd into a range of lesions in Alzheimer's disease. $J$ Neuropathol Exp Neurol 46:611-622.

Khachaturian ZS (1985) Diagnosis of Alzheimer's disease. Arch Neurol 42:1097-1105.

Kowall N, Kosik KS (1987) Axonal description and aberrant localization of tau protein characterized the neurofibrillary pathology of Alzheimer disease. Ann Neurol 22:639-643.

Laemmli UK (1970) Cleavage of structural proteins during the assembly of the head of the bacteriophage T4. Nature 227:680-685.

Lindwall G, Cole RD (1984) The purification of tau protein and the occurrence of two phosphorylation states of tau in brain. J Biol Chem 259:12231-12245.

Manetto V, Perry G, Tabaton M, Mulvihill P, Fried V, Smith H, Gambetti $P$, Autilio-Gambetti $L$ (1988) Ubiquitin is associated with abnormal cytoplasmic filaments in neurodegenerative diseases. Proc Natl Acad Sci USA 85:4501-4505.

McKee AC, Kowall NW, Kosik KS (1989) Microtubular reorganization and dendritic growth response in Alzheimer's disease. Ann Neurol 26:652-659.

McKee AC, Kosik KS, Kowall NW (1990) Dystrophic neurites in the neocortex are the critical neuropathological correlate of dementia in Alzheimer's disease. J Neuropathol Exp Neurol 49:336.

Metuzals J, Robitaille Y, Houghton S, Gauthier S, Leblanc R (1987) Transformation of the neurofilamentous network components into PHF-like strands and PHF paracrystals. In: Alterations in the neuronal cytoskeleton in Alzheimer disease (Perry G, ed), pp 75-95. New York: Plenum.

Metuzals J, Robitaille Y, Houghton S, Gauthier S, Yang CY, Leblanc R (1988) Neuronal transformations in Alzheimer's disease. Cell Tissue Res 252:239-248.

Ohta M, Marceau N, Perry G, Manetto V, Gambetti P, Autilio-Gambetti L, Metuzals J, French SW (1988) Relationship of the Mallory body to the ubiquitin system. Lab Invest 59:848-856.

Onorato M, Mulvihill P, Connolly J, Galloway P, Whitehouse P, Perry $G$ (1989) Alteration of neuritic cytoarchitecture in Alzheimer disease. In: Alzheimer's disease and related disorders (Iqbal K, Wisniewski HM, Winblad B, eds), pp 781-789. New York: Liss.

Perry G, Brinkley BR, Bryan J (1982) Interaction of calcium-calmodulin in microtubule assembly in vitro. In: Muscle and cell motility Vol 2 (Dowben RM, Shay JW, eds), pp 73-82. New York: Plenum.

Perry G, Mulvihill P, Manetto V, Autilio-Gambetti L, Gambetti P (1987) Immunocytochemical properties of Alzheimer straight filaments. J Neurosci 7:3736-3738.

Sternberger LA (1986) Immunocytochemistry, 3d ed. New York: Wiley. 
Tabaton M, Whitehouse PJ, Perry G, Davies P, Autilio-Gambetti L Gambetti P (1988) Alz-50 recognizes abnormal filaments in Alzheimer's disease and progressive supranuclear palsy. Ann Neurol 24: 407-413.

Tabaton M, Mandybur T, Perry G, Onorato M, Autilio-Gambetti L, Gambetti P (1989) The widespread alternation of neurites in Alz- heimer disease may be unrelated to amyloid deposition. Ann Neurol 26:771-778.

Towbin H, Staehelin T, Gordon J (1979) Electrophoretic transfer of proteins from polyacrylamide gels to nitrocellulose sheets: procedure and some applications. Proc Natl Acad Sci USA 76:4350-4354. 